\title{
Pilot Balloon Observations at Mauritius*
}

\begin{abstract}
THE study of the upper wind currents over Mauritius with the aid of pilot balloons was begun in July 1925, and the results obtained in the early years have been discussed by Walter and McCurdy (Geophysical Memoir No. 39 of the British Meteorological Office and Miscellaneous Publications of the Royal Alfred Observatory, No. 9 are two of the papers dealing with this subject). In Part 1 of the memoir under review is summarised all the ascents from July 1928 until December 1929 in tables giving the speed and direction of the wind mostly for steps of 500 or 1,000 metres height; and also, in the form of monthly means and frequencies, the results of recent and earlier ascents. As many as 28 ascents have reached a height of 8,000 metres and 80 a height of 6,000 metres ; the general summary is taken up to 8,000 metres.
\end{abstract}

In Part 2 the results are discussed. This discussion is much more thorough than is usual in such work. It is an endeavour to "build up a scheme of the upper wind structure within the tropics and show how the scheme is modified by the seasons", to quote the authors' own words. In it they remark that meteorologists appear to be diffident in applying the geostrophic wind formula-which covers the case of air motion along a great circle-to regions within the tropics, and that their own experience has suggested to them that the isobaric chart between latitudes $5^{\circ}$ and $25^{\circ} \mathrm{S}$. is normally composed of "straight isobars", to which the geostrophie formula applies, and that the observed winds fit the isobars "at least as well, both in speed and direction, in latitude $5^{\circ}$ as in latitude $50^{\circ}$ ",

They then proceed to derive from the geostrophic formula an expression for the height of the reversal layer between the lower easterly and the upper

* Miscellaneous Publications of the Royal Alfred Observatory, No. 11. "Pilot Balloon Observations at Mauritius." By R. A. Watson and N. R. McCurdy. Pp. $17+3$ plates. (Mauritius.) westerly wind currents over that part of the tropics that lies more than $5^{\circ}$ from the equator. The formula obtained is as follows:

$$
-\frac{H}{67 \cdot 4} \cdot \frac{P_{0}}{\left(T_{h}\right)^{2}} \cdot \frac{d T_{h}}{d \lambda}=\frac{d P_{0}}{d \lambda}
$$

where $H$ is the height of the reversal layer in metres, $T_{h}$ is mean temperature of the air from the surface to height $H$, in degrees Centigrade absolute, $\lambda$ is latitude, and $P_{\theta}$ is pressure at sea-level, in millibars.

The values given by this equation after various plausible assumptions such as the constancy of mean horizontal temperature gradients at all heights (based on the known smallness of horizontal temperature gradient at the surface over the tropical oceans, and the probability that lapse-rate varies very little) range from 17,700 metres in latitude $10^{\circ}$ to 3,200 metres in latitude $20^{\circ}$.

The authors compare the theoretical figures with those found over various tropical islands, for example at Apia, Guam and Honolulu, and allowing for the seasonal disturbance caused by the shifting of the thermal equator with varying declination of the sun, obtain fair agreement on the whole, though the figures are not applicable in the neighbourhood of large land areas. The work concludes with diagrams showing the observed seasonal variation in the height of the reversal layer over Mauritius, monthly means of velocity up to 8,000 metres, and wind roses up to the same height.

The mathematical analysis in this memoir may possibly be open to criticism here and there, but a serious attempt to go beyond the descriptive stage and to relate local conditions with the general atmospheric circulation is rarely made in papers dealing with upper winds, and is particularly welcome when the region studied forms part of an immense area for which few meteorological data relating to such winds are available.

\section{Sexual Cycle in the Rhesus Monkey}

$\mathrm{D}_{\text {of }}^{\mathrm{n}}$ . CARL G. HARTMANN has recently published* an account of his detailed investigation of menstruation and pregnancy in the monkey, Macacus rhesus. Corner's generalisations concerning the cellular content of the vagina of the monkey are fully corroborated; the leucocyte number falls near the middle of the cycle and the number of cornified cells rises to a peak near the end of the interval. Instead of the vaginal smear method, vaginal lavage has been adopted and has proved more instructive since it enables the cells to be studied in the living condition, vitally stained with methylene blue, which also affords a ready means of differentiating the kinds of cornified cells in the vaginal lumen. The amount of desquamation from the vaginal wall can be quickly read off, after lavage, in terms of the percentage of sediment in the collecting tube. Because of the constancy and reliability of the curve of vaginal desquamation as compared with the character of the cornified cells and leucocytes recovered, the latter are no longer studied and recorded; the amount of desquamation and of uterine bleeding are

* Carnegie Inst. of Washington: Contributions to Fmbryology, vol. 23, No. 134, August, 1932 . the two principal factors now studied in menstruating females. When the vagina is practically free of desquamated cells the animal is at a low ebb sexually, and the uterus and ovaries are small and hypofunctional.

Forty-two females, mostly full grown, have furnished data for more than seven hundred menstrual cycles-from a few to fifty-one for the individual females. The average of four hundred cycles for twenty-two of the more vigorous females is twentyseven to twenty-eight days, but the variations are wide. The average duration of flow approaches four to six days. Dr. Hartmann's paper adds to the literature several hundred proven cases of menstruation without ovulation; the same individual, with regular menstrual rhythm, may alternate ovulatory with non-ovulatory cycles. From an inspection of the ovaries in summer and in winter it was found that the almost complete sterility of the female Rhesus in the warm months of the year was due to her failure to ovulate. A tentative estimate of the age of puberty (the time of the first menstruation) and of maturity (the capacity to ovulate and conceive) is three and four-and-a-half years respectively. 